of poly-drug abuse this is a serious gap in intelligence.

P. H. Connell, ${ }^{15}$ in furnishing a very complete description of the British system and the thinking which lay behind the design of that system, has put emphasis on the need for careful epidemiological and sociological research. It is much to be desired that the Anglo-American debate will increasingly be fed by hard facts, and while eschewing the riskier type of inference we may surely hope that each country will learn considerably from the other's still evolving experience.

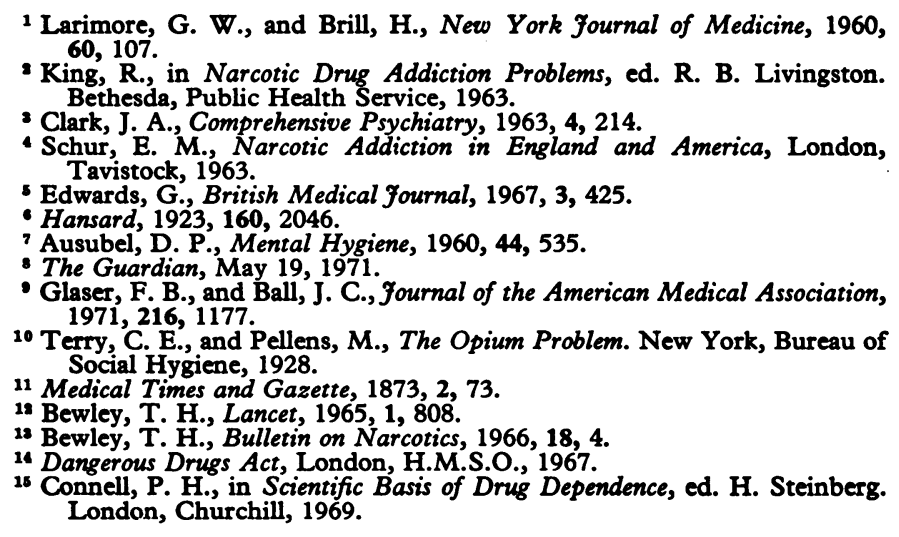

\section{Atypical Gonorrhoea}

The increase in cases of gonorrhoea throughout the world has been accompanied by more frequent recognition of a relatively mild form of metastatic infection which seems to be difficult to diagnose. The salient characteristics of this condition have been described in the B.M.f. from time to time, ${ }^{12}$ but the fact that the correct diagnosis is so frequently missed in the early stages makes them worthy of repetition.

The syndrome occurs more often in women than in men, presumably because uncomplicated gonorrhoea is often symptomless or difficult to diagnose in women, and treatment is therefore delayed. It is apt to present as a triad of fever; polyarthritis, which may be migratory; and lesions of the skin. The fever is usually moderate and may begin with one or more rigors. The arthritis may affect large or small joints and joints of the arms or legs. Effusions may or may not be present and gonococci have been isolated from the joint fluid in some cases. The rashes appear to be of two kinds. Firstly there may be papulopustular lesions, which tend to be widely scattered and are often situated on the extremities near to joints. Sometimes these become vesicular or pustular lesions which may show haemorrhagic centres. The gonococcus has been grown from the contents of vesicles or pustules in some cases. On healing, the papules tend to become crusted. The second kind of rash is urticarial or one resembling erythema nodosum.

In a few patients with atypical gonorrhoea the gonococcus has been grown in blood culture. The patient usually has undiagnosed genital gonorrhoea and may have signs of salpingitis. The response to systemic penicillin is prompt and satisfactory. J. K. Wheeler and his colleagues ${ }^{3}$ describing five cases in women, pointed out that the appearance and course of the manifestations are so variable that there might be a considerable delay before the pos- sibility of gonorrhoeal infection was even considered-as indeed happened in two of their cases. These patients are usually referred to the general physician, rheumatologist, or dermatologist, rather than the venereologist or gynaecologist, who might more readily suspect the possibility of venereal infection. The arthritis may suggest rheumatic fever, drug reaction, serum sickness, rheumatoid arthritis, systemic lupus erythematosus, Reiter's disease, or various other conditions. The rash may be mistaken for erythema multiforme, especially the bullous type, surface infection with ordinary cocci, or erythema nodosum.

Probably the atypical gonorrhoea syndrome will be seen more frequently in future, and all those in general or specialist practice who may see these patients should be alert to the possibility. Thorough investigation of the usual sites of gonococcal infection is essential whenever suspicion arises. Moreover, the correct diagnosis will be made much easier if the doctor has access to a supply of the right culture medium for growing the gonococcus and also to a laboratory which can undertake fluorescent antibody studies for detecting the organism.

1 Wolf, C. B., Goodman, H. V., and Vahrman, J., British Medical fournal, $1970,1,271$

British Medical fournal, 1970, 3, 420

Wheeler, J. K., Heffron, W. A., and Williams, R. C. jun., American fournal of Medical Sciences, 1970, 260, 150.

\section{Pulmonary Candidiasis in Infants}

Pulmonary candidiasis was first described by A. Castellani ${ }^{1}$ in 1912 in adults, and several cases have been reported since. In older children the disease almost always occurs after prolonged antibiotic therapy. The incidence in infancy is very low despite the frequency of oral and other forms of thrush.

This rarity of pulmonary infections is attributed to a natural resistance of columnar epithelium to invasion by the candida, which is particularly prone to attack stratified epithelium. In 1962 B. Emanuel and his colleagues ${ }^{2}$ described two cases in newborn infants seen during a period of 13 years. They reviewed the literature and found till then only 15 authenticated cases. All these occurred sporadically. Later A. Linhartova and W. Chung ${ }^{3}$ found a $1 \%$ incidence among 1,000 necropsies carried out on newborn infants. However, recently U. D. Koenig ${ }^{4}$ found five fatal instances within a few months among 38 necropsies carried out on infants and children. Unless this was a remarkable chance, the condition may be occurring more often than it is diagnosed. This is all the more likely because the illness is not necessarily fatal and its diagnosis in life is most difficult.

The predisposing causes are infections of the maternal birth canal and the free but not necessarily wise prophylactic or therapeutic administration of antibiotic drugs to newborn and especially premature babies. ${ }^{5}$ In the majority of patients oral or less commonly oesophageal thrush was present, but it was not invariably so even in pathologically proved cases. The correct diagnosis can rarely be made with certainty in life, partly because the pneumonic illness has no distinguishing clinical or radiological features and partly because the isolation of candida from the mouth or pharynx is no proof of the diagnosis of pulmonary candidiasis. The 